\title{
Patient-controlled oral analgesia for postoperative pain management following total knee replacement
}

\author{
Patti Kastanias RNEC MScA, Sue Gowans BScPT PhD, Paul S Tumber MD FRCPC, \\ Kianda Snaith RN MScN APRN, Sandra Robinson RNEC MN
}

\author{
P Kastanias, S Gowans, PS Tumber, K Snaith, S Robinson. Patient- \\ controlled oral analgesia for postoperative pain management \\ following total knee replacement. Pain Res Manage 2010;15(1):11- \\ 16.
}

PURPOSE: To investigate whether patient-controlled oral analgesia (PCOA) used by individuals receiving a total knee replacement could reduce pain, increase patient satisfaction, reduce opioid use and/or reduce opioid side effects when compared with traditional nurse (RN)-administered oral analgesia.

METHODS: Patients who underwent an elective total knee replacement at a quaternary care centre (Toronto Western Hospital, Toronto, Ontario) were randomly assigned to either PCOA or RN-administered short-acting oral opioids on postoperative day 2. Subjects in the RN group called the $\mathrm{RN}$ to receive their prescribed short-acting opioid. Subjects in the PCOA group kept a single dose of their prescribed oral opioid at their bedside and took this dose when they felt they needed it, to a maximum of one dose every $2 \mathrm{~h}$. Study outcomes, collected on postoperative day 2, included pain (measured by the Brief Pain Inventory - Short Form), patient satisfaction (measured by the Pain Outcome Questionnaire Satisfaction subscale - component II), opioid use (oral morphine equivalents), opioid side effects (nausea, pruritis and/or constipation) and knee measures (maximum passive knee flexion and pain at maximum passive knee flexion, performed on the operative knee).

RESULTS: Study outcomes were analyzed twice. First, for a subset of 73 subjects who remained in their randomly assigned group (PCOA group, $\mathrm{n}=36$; $\mathrm{RN}$ group, $\mathrm{n}=37$ ), randomized analyses were performed. Second, for the larger sample of 88 subjects who were categorized by their actual method of receiving oral opioids (PCOA group, $n=41 ; R N$ group, $n=47$ ), as-treated analyses were performed. There were no differences in study outcomes between the PCOA and RN groups in either analysis.

CONCLUSION: PCOA was not superior to RN administration on study outcomes. However, PCOA did not increase opioid use or pain. PCOA remains an important element in the patient-centred care facility.

Key Words: Arthroplasty; Knee replacement; Oral administration; Patientcontrolled analgesia; Randomized clinical trials

\section{L'analgésie orale contrôlée par les patients pour la prise en charge de la douleur après une arthroplastie totale du genou}

OBJECTIF : Examiner si l'analgésie orale contrôlée par les patient (AOCP) utilisée par les personnes qui subissent une arthroplastie totale du genou peut réduire la douleur, accroître la satisfaction du patient, réduire le recours aux opiacés ou les effets secondaires des opiacés par rapport à une analgésie orale classique administrée par une infirmière.

MÉTHODOLOGIE : Les patients qui ont subi une arthroplastie totale du genou non urgente à un centre de soins quaternaires (Toronto Western Hospital de Toronto, en Ontario) ont été répartis au hasard entre une AOCP ou des opiacés oraux à action brève administrés par une infirmière le deuxième jour postopératoire. Les sujets du groupe traité par l'infirmière l'appelaient pour recevoir leur opiacé à action brève prescrit, tandis que le groupe de patients sous AOCP conservaient une dose unique d'opiacé oral prescrit à leur chevet et la prenaient lorsqu'ils en ressentaient le besoin, jusqu'à concurrence d'une dose aux deux heures. Les issues de l'étude, colligées le deuxième jour postopératoire, incluaient la douleur (mesurée selon le bref inventaire de la douleur - court), la satisfaction du patient (mesurée selon la sous-échelle de satisfaction du questionnaire d'issue de la douleur - élément II), le recours aux opiacés (équivalents oraux de la morphine), les effets secondaires des opiacés (nausées, prurit ou constipation) et les mesures du genou (flexion passive maximale du genou et douleur lors de la flexion passive maximale du genou opéré).

RESULTATS : Les chercheurs ont analysé les issues de l'étude deux fois. D'abord, dans un sous-groupe de 73 sujets demeurés dans leur groupe attribué au hasard (groupe sous AOCP, n=36; groupe traité par l'infirmière, $\mathrm{n}=37$ ), ils ont effectué des analyses randomisées. Ensuite, au sein du plus grand échantillon de 88 sujets classés selon la méthode réelle d'administration des opiacés (groupe sous AOCP, $n=41$; groupe traité par l'infirmière, $n=47$ ), ils ont procédé à des analyses selon le traitement. Dans les deux analyses, ils n'ont constaté aucune différence d'issues entre le groupe sous AOCP et celui traité par l'infirmière.

CONCLUSION : L'AOCP n'était pas supérieure à l'administration par l'infirmière pour les issues de l'étude. Cependant, l'AOCP n'accroissait pas l'utilisation des opiacés ou la douleur. L'AOCP demeure un élément important dans l'établissement de soins axés sur les patients.

self-titrate IV opioid medication and, thus, actively participate in their own postoperative pain management. Meta-analyses indicate that, compared with traditional, as-needed, nurse (RN)-administered dosing, IV PCA provides better pain control and greater patient satisfaction (6). However, after IV PCA is discontinued, postoperative pain is commonly managed with RN-administered, as-needed, oral opioid medication. This means that patients are no longer in control of their own pain. Instead, they must rely on the $\mathrm{RN}$ to respond to their call bell, withdraw the medication from the medication (TKR) surgery, including the use of intravenous (IV) patientcontrolled analgesia (PCA) (5). IV PCA allows patients to 


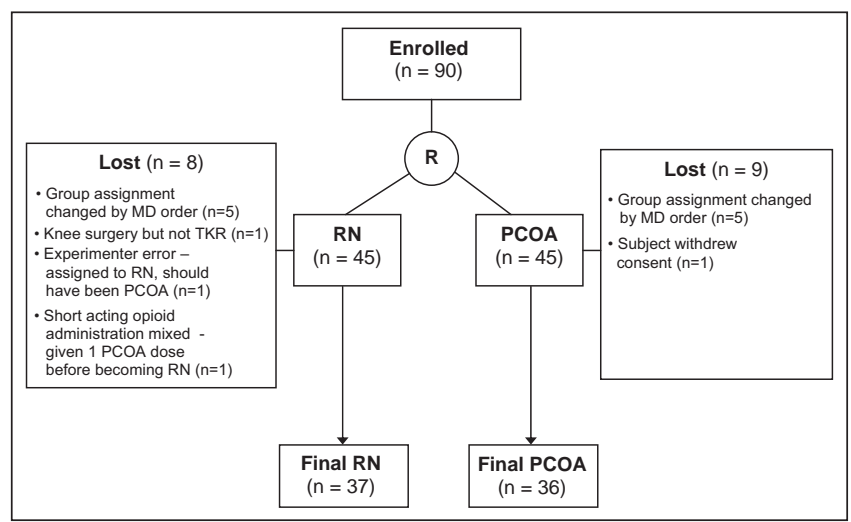

Figure 1) Randomized analysis of subjects. MD Doctor; PCOA Patient-controlled oral analgesia group; $R$ Random assignment; $R N$ Nurse-administered oral analgesia group; TKR Total knee replacement

dispenser and then administer the medication - often at the expense of a time delay and increasing pain.

Patient-controlled oral analgesia (PCOA) is a relatively new approach to pain management that puts the control of short-acting oral medications for postoperative pain back in the hands of the patient. Only a few randomized controlled studies $(7,8)$ have evaluated PCOA using specialized delivery systems. In these studies, liquid morphine was dispensed from a specially constructed reservoir via modified IV tubing. These early PCOA studies $(7,8)$ suggested that the benefits of PCOA were similar to IV PCA; that is, they led to increased patient satisfaction and better pain control.

For the past six years, we have used a simpler protocol for PCOA. In our PCOA protocol, there is no specialized dispenser. Instead, patients keep a single dose of a short-acting opioid secured in a child-resistant vial at their bedside for use when required (9). The present study evaluated the effectiveness of this simpler method of PCOA for pain control. The primary hypothesis of the study was that PCOA subjects would have less average pain in the preceding $24 \mathrm{~h}$, as measured by the Brief Pain Inventory - Short Form (BPI-SF) (ie, better pain control). The secondary hypotheses were that our simpler version of PCOA would improve patient satisfaction, reduce oral opioid use, lessen opioid side effects, and/or improve knee flexion and knee pain.

\section{Subjects}

\section{METHODS}

Subjects were inpatients in a single orthopedic ward at a quaternary care facility (Toronto Western Hospital, Toronto, Ontario) between November 2004 and November 2005. To be included in the present study, subjects had to have undergone a primary or secondary elective TKR; be able to independently open a child-resistant vial (ie, have adequate manual strength and dexterity, and visual and mental acuity to do so); and be proficient in English (both oral and written). Subjects were excluded from the study if they had a history of substance abuse, a major psychiatric disorder or sleep apnea; had episodes of respiratory depression during the current admission; were confused or disoriented; or were pregnant. The University Health Network Research Ethics Board approved the present study and all subjects provided informed, written consent.

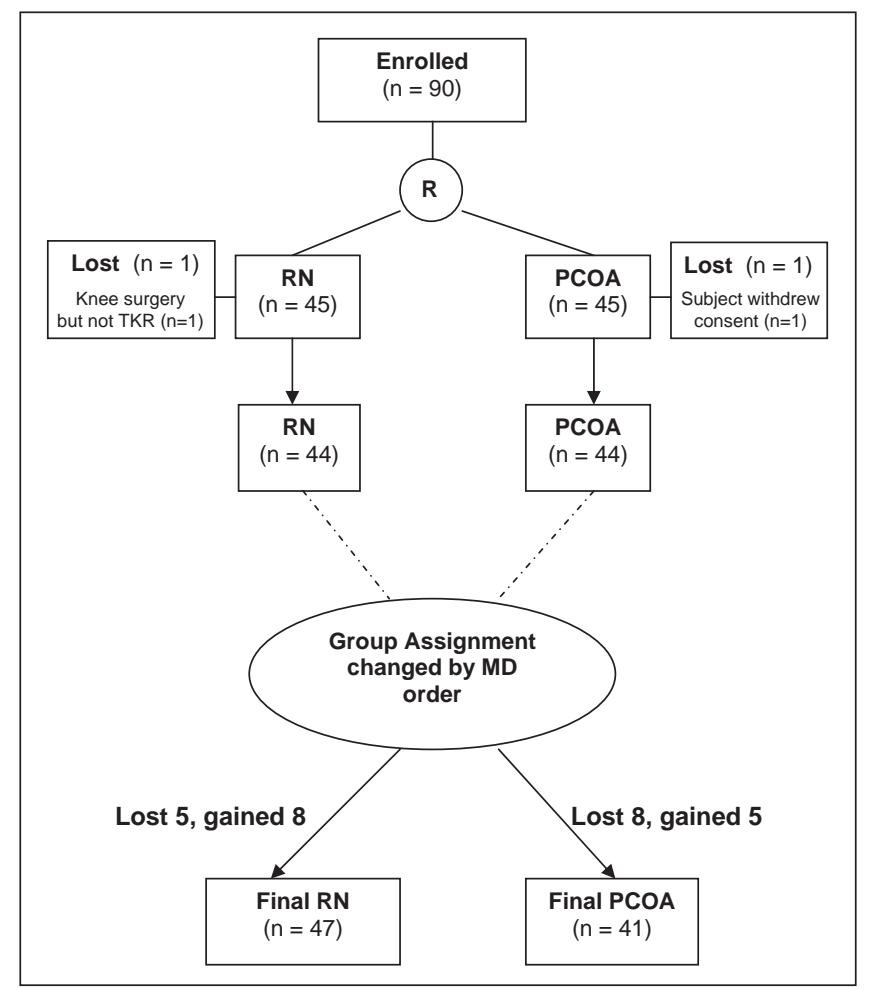

Figure 2) As-treated analysis of subjects. MD Doctor; PCOA Patient-controlled oral analgesia group; $R$ Random assignment; $R N$ Nurse-administered oral analgesia group; TKR Total knee replacement

\section{Design}

On the morning of postoperative day (POD) 2, when IV PCA was routinely discontinued, subjects were randomly assigned, using a random numbers table, to one of two oral analgesia administration groups: a $\mathrm{RN}$-administered group or a PCOA group. The acute pain service (APS) physician or advanced practice nurse wrote the relevant clinical order (for $\mathrm{RN}$-administered or patient-controlled short-acting opioids) as per their random group assignment. Subjects' group assignments dictated only the means by which they received short-acting oral opioids (RN or patient controlled). Subjects in both groups received their around-the-clock, long-acting opioids from their RN, when prescribed by the APS. The APS determined the type and dose of short-acting opioid for each study subject via patient assessment. The APS monitored pain control of all subjects via daily pain rounds as per standard clinical practice at the facility; the APS was accessible by pager $24 \mathrm{~h}$ per day in case reassessment was required. Study outcomes were assessed for the first day of RN-administered or PCOA short-acting opioids (ie, POD 2). A longer period of data collection was intended, but not achievable, because shortly after the present study was launched, the facility began to promote fast-track or early hospital discharge by POD 3, versus the previous practice of discharge on POD 5 for individuals after TKR.

\section{PCOA protocol}

PCOA subjects received a single dose of their individually prescribed, short-acting opioid that was kept in a childresistant vial at their bedside. PCOA subjects were instructed to take this bedside dose when they felt the need to do so, to a maximum of one dose every $2 \mathrm{~h}$. After they had taken a dose, 
TABLE 1

Demographic and clinical characteristics of study sample

\begin{tabular}{|c|c|c|c|c|c|c|}
\hline \multirow[b]{2}{*}{ Characteristic } & \multicolumn{3}{|c|}{ Randomized analysis } & \multicolumn{3}{|c|}{ As-treated analysis } \\
\hline & RN (n=37) & PCOA $(n=36)$ & $\mathbf{P}$ & RN (n=47) & PCOA $(n=41)$ & $\mathbf{P}$ \\
\hline Age, years, mean $\pm \mathrm{SD}$ & $66.3 \pm 9.4$ & $64.8 \pm 9.3$ & 0.477 & $66.7 \pm 9.3$ & $64.4 \pm 9.8$ & 0.265 \\
\hline \multicolumn{7}{|l|}{ Surgery, \% } \\
\hline Primary total knee replacement & 95 & 94 & 0.978 & 94 & 95 & 0.761 \\
\hline Secondary total knee replacement & 5 & 6 & & 6 & 5 & \\
\hline General & 30 & 28 & 0.854 & 28 & 24 & 0.592 \\
\hline Spinal & 70 & 72 & & 70 & 76 & \\
\hline Spinal and epidural & 0 & 0 & & 2 & 0 & \\
\hline \multicolumn{7}{|l|}{ Perioperative nerve blocks, $\%$ yes } \\
\hline Sciatic & 81 & 78 & 0.727 & 81 & 81 & 0.966 \\
\hline Femoral & 100 & 97 & 0.307 & 96 & 98 & 0.640 \\
\hline Morphine and acetaminophen/codeine $30 \mathrm{mg}$ & 0 & 0 & & 2 & 0 & \\
\hline Oxycodone only & 32 & 31 & & 30 & 37 & \\
\hline Oxycodone and morphine (intravenous) & 3 & 0 & & 2 & 0 & \\
\hline Oxycodone and hydromorphone & 0 & 0 & & 2 & 0 & \\
\hline Hydromorphone only & 11 & 11 & & 13 & 10 & \\
\hline Hydromorphone (oral and intravenous) & 0 & 3 & & 0 & 2 & \\
\hline Acetaminophen/oxycodone $5 \mathrm{mg}$ & 3 & 0 & & 2 & 0 & \\
\hline $\begin{array}{l}\text { Acetaminophen/oxycodone } 5 \mathrm{mg} \text {, oxycodone and } \\
\text { hydromorphone }\end{array}$ & 0 & 0 & & 0 & 2 & \\
\hline Nil & 0 & 0 & & 2 & 0 & \\
\hline \multicolumn{7}{|l|}{ Long-acting opioids } \\
\hline
\end{tabular}

*Oral administration unless otherwise indicated. PCOA Patient-controlled oral analgesia group; RN Nurse-administered oral analgesia group

they were instructed to use their bedside bell to call nursing staff to refill the vial with another single dose. The protocol was that nursing staff should refill a PCOA vial within $30 \mathrm{~min}$ of a request to do so.

\section{Study outcomes}

The primary outcome was average pain severity in the previous $24 \mathrm{~h}$, as measured by a subscale of the BPI-SF. The BPI-SF is a validated tool that has been widely used in various populations and settings, including acute care $(10,11)$. The BPI-SF measures pain severity, pain relief and pain interference with various activities of daily living and mood. For completeness, all of the aforementioned subscales of the BPI-SF are reported.

There were four secondary study outcomes: the Pain Outcome Questionnaire (POQ) Satisfaction subscale - component II (12); oral opioid use (oral morphine equivalents); medication use for nausea, pruritis and/or constipation (a proxy measure for oral opioid side effects); and operated knee measures - maximum passive knee flexion and pain at maximum passive knee flexion. The Satisfaction subscale of the POQ evaluated patients' satisfaction with the RN's treatment, percentage pain relief, need for more medication, interference with sleep and wait time for medication. Oral opioid use until 24:00 on POD 2 was extracted from the facility's electronic patient record (EPR), maintained as a separate record for each admission of each patient, and expressed in oral morphine equivalents. For PCOA subjects only, short-acting opioid use was corrected by calculating the sum of all short-acting doses (in oral morphine equivalents) for POD 2 from the EPR minus the first dose on POD 2. Subtracting the first dose for PCOA subjects was necessary to correct for the fact that PCOA subjects kept a single short-acting dose at their bedside for their subsequent use and, therefore, by protocol, had one unused dose of short-acting opioid at the end of the $24 \mathrm{~h}$ period. Due to limited funds for the present study, opioid side effects were not tracked directly; they were inferred by tracking the administration of medication for nausea, pruritis and/or constipation from the EPR documentation. At the facility, all TKR patients have standard, as-needed medication orders for nausea (dimenhydrinate; if dimenhydrinate is ineffective after two doses, then granisetron is used), pruritis (diphenhydramine) and constipation (bisacodyl suppository if no bowel movement for 
TABLE 2

Brief Pain Inventory - Short Form (BPI-SF) and Pain Outcome Questionnaire (POQ) Satisfaction subscale - component II scores

\begin{tabular}{|c|c|c|c|c|c|c|}
\hline & \multicolumn{3}{|c|}{ Randomized analysis } & \multicolumn{3}{|c|}{ As-treated analysis } \\
\hline & $\mathrm{RN}(\mathrm{n}=37)$ & PCOA $(n=36)$ & $\mathbf{P}$ & RN (n=47) & PCOA $(n=41)$ & $\mathbf{P}$ \\
\hline \multicolumn{7}{|l|}{ BPI-SF, mean \pm SD } \\
\hline Worst pain in last $24 \mathrm{~h}$ & $7.6 \pm 2.4$ & $7.0 \pm 2.1$ & 0.329 & $7.2 \pm 2.6$ & $7.1 \pm 1.9$ & 0.833 \\
\hline Least pain in last $24 \mathrm{~h}$ & $2.0 \pm 1.9$ & $1.9 \pm 1.6$ & 0.953 & $2.0 \pm 1.8$ & $2.0 \pm 1.8$ & 0.868 \\
\hline Average pain in last $24 \mathrm{~h}$ & $4.3 \pm 1.9$ & $4.3 \pm 1.7$ & 0.996 & $4.2 \pm 1.9$ & $4.2 \pm 1.7$ & 0.980 \\
\hline Current pain & $3.3 \pm 2.5$ & $2.6 \pm 2.3$ & 0.291 & $3.1 \pm 2.3$ & $3.0 \pm 2.5$ & 0.833 \\
\hline Amount of pain relief & $71.3 \pm 23.9$ & $64.6 \pm 27.5$ & 0.332 & $73.2 \pm 22.2$ & $63.5 \pm 26.3$ & 0.104 \\
\hline Total pain interference & $34.3 \pm 14.0$ & $30.0 \pm 12.7$ & 0.230 & $30.9 \pm 15.2$ & $32.3 \pm 13.6$ & 0.695 \\
\hline \multicolumn{7}{|l|}{ POQ - Satisfaction subscale, \% } \\
\hline \multicolumn{7}{|l|}{ How satisfied were you with the way your nurse treated your pain? } \\
\hline Very satisfied & 77 & 78 & 0.665 & 78 & 75 & 0.830 \\
\hline Moderately satisfied & 21 & 22 & & 17 & 22 & \\
\hline Slightly satisfied & 0 & 0 & & 0 & 0 & \\
\hline Dissatisfied & 3 & 0 & & 5 & 3 & \\
\hline Very dissatisfied & 0 & 0 & & 0 & 0 & \\
\hline $\begin{array}{l}\text { How much relief did you obtain from pain treatments or medication? } \\
\text { (\% relief) }\end{array}$ & $71.4 \pm 27.3$ & $68.9 \pm 25.9$ & 0.720 & $73.1 \pm 25.4$ & $66.1 \pm 27.8$ & 0.274 \\
\hline $\begin{array}{l}\text { Was there a time the medication didn't help and you asked for more or } \\
\text { something different? (\% yes) }\end{array}$ & 46 & 23 & 0.069 & 42 & 32 & 0.424 \\
\hline $\begin{array}{l}\text { What percentage of time did unrelieved pain interfere with your sleep? } \\
(\text { mean } \pm S D)\end{array}$ & $26.2 \pm 28.7$ & $29.6 \pm 28.1$ & 0.649 & $24.3 \pm 27.0$ & $33.3 \pm 29.7$ & 0.193 \\
\hline
\end{tabular}

PCOA Patient-controlled oral analgesia group; RN Nurse-administered oral analgesia group

TABLE 3

Medications for opioid side effects and operative knee outcomes

\begin{tabular}{|c|c|c|c|c|c|c|}
\hline & \multicolumn{3}{|c|}{ Randomized analysis } & \multicolumn{3}{|c|}{ As-treated analysis } \\
\hline & RN (n=37) & $\operatorname{PCOA}(n=36)$ & $\mathbf{P}$ & RN (n=47) & $\operatorname{PCOA}(n=41)$ & $\mathbf{P}$ \\
\hline \multicolumn{7}{|l|}{ Medications for opioid side effects, $\%$} \\
\hline Nausea* & 13.5 & 19.4 & 0.494 & 14.9 & 19.5 & 0.565 \\
\hline Pruritis $^{\dagger}$ & 13.5 & 2.8 & 0.095 & 14.9 & 4.9 & 0.122 \\
\hline Constipation $^{\ddagger}$ & 16.2 & 5.6 & 0.145 & 12.8 & 4.9 & 0.199 \\
\hline Maximum knee flexion (passive), mean $\pm \mathrm{SD}$ & $74.8 \pm 13.9$ & $75.8 \pm 13.2$ & 0.781 & $75.5 \pm 14.2$ & $76.5 \pm 12.8$ & 0.779 \\
\hline Pain at maximum knee flexion (passive), mean \pm SD & $5.4 \pm 2.4$ & $6.5 \pm 2.3$ & 0.176 & $5.6 \pm 2.5$ & $6.4 \pm 2.2$ & 0.296 \\
\hline
\end{tabular}

Maximum knee flexion measured in degrees. Pain at maximum knee flexion measured on a numerical rating scale, where $0=$ no pain and $10=$ worst pain imaginable. *Medication included dimenhydrinate, granisetron, metoclopramide and prochlorperazine; ${ }^{\dagger}$ Medication included diphenhydramine; ${ }^{\ddagger}$ Medication included bisacodyl suppository and sodium phosphate enema. PCOA Patient-controlled oral analgesia group; RN Nurse-administered oral analgesia group

three days; sodium phosphate enema if bisacodyl suppository is ineffective). Opioid side effects were deemed present if subjects' individual online drug records documented that they were given any of these standard, as-needed medications on POD 2 or if they were given metoclopramide and/or prochlorperazine for nausea on POD 2 (these drugs could be individually ordered for patients). Standard preordering of side effect drugs reduced, but did not eliminate, a type 2 error (coding no side effect when there was a side effect) because medications were available immediately on patient need.

\section{Analyses}

Demographic, clinical characteristics and study outcomes were compared between RN and PCOA groups using $\chi^{2}$ tests for categorical variables and two-tailed independent $t$ tests for continuous variables. Data were analyzed twice - once for subjects who were randomly assigned and remained in their randomly assigned group (randomized analyses), and again for all subjects (those who remained in their randomly assigned group and those who did not) according to their actual method of oral opioid administration (as-treated analyses). Analyzing data two ways allowed retention of the data of subjects who had their method of short-acting opioid administration changed after their random assignment for the following reasons: their supervising physician's preference superseded their random assignment; personal factors of the subject (eg, the subject was subsequently judged as being unable to open the bedside vial or was unable in some other way to comply with the PCOA protocol); or for any other reason. Statistical significance for all analyses was set at $\mathrm{P} \leq 0.05$. Unless otherwise stated, variability is expressed as SD. Sample size calculations (based on a 2.0 difference in the average pain subscore of the BPI-SF, a 20\% dropout rate, and alpha $=0.05$ and beta $=0.8$ ) suggested 36 subjects per group were required for statistical significance.

\section{RESULTS}

Subject allocation is shown for both randomized and as-treated analyses in Figures 1 and 2. Briefly, 90 subjects were randomly assigned to the PCOA $(n=45)$ and RN $(n=45)$ groups. Two subjects were subsequently excluded from all analyses (one 
PCOA group subject withdrew consent and one RN group subject had knee surgery but not a TKR). Thus, as-treated analyses were based on 88 subjects and randomized analyses were based on 73 subjects. Thirteen additional subjects were excluded from randomized analyses because they had their randomly assigned group changed by the doctor's order before they received their oral opioid medications (five RN group and eight PCOA group subjects). The reasons for changing the randomly assigned group could be verified using chart review for only two PCOA subjects: postoperative confusion $(n=1)$ and lack of manual dexterity $(n=1)$. A further two subjects were excluded from randomized analyses because of experimenter error: one subject was incorrectly recorded as being randomly assigned to the $\mathrm{RN}$ group when he or she was actually randomly assigned to the PCOA group, and drug administration was mixed for one $\mathrm{RN}$ group subject, who received a single PCOA dose (by the doctor's order) before receiving an $\mathrm{RN}$-administered dose. This subject was treated as an $\mathrm{RN}$ group subject for the as-treated analyses.

Subjects' demographic and clinical care characteristics (length of stay, surgery, type of anesthesia, use of perioperative nerve blocks, and type of short-and long-acting opioids) are summarized in Table 1 . As Table 1 indicates, there were no significant differences between groups on demographic or clinical care characteristics for any analysis. There were also no group differences in subjects' scores on the BPI-SF or the POQ Satisfaction subscale (Table 2). The frequency of opioid side effects and subjects' oral opioid use for POD 2 are summarized in Table 3 and Figure 3, respectively. There were no significant group differences for oral opioid use or opioid side effects on POD 2.

\section{DISCUSSION}

The present study failed to detect any difference in pain control between PCOA and RN administration of oral opioids. There were also no significant differences in subjects' satisfaction, oral opioid use or opioid side effects.

We may have failed to detect a difference between PCOA and $\mathrm{RN}$-administered groups because we studied the impact of the method of administration for only a brief period of time on POD 2, when pain control was changed from IV PCA to oral opioids. This brief period of data collection was unexpected. Our original intent was to collect study outcomes from POD 2 to POD 4, but the introduction of an early discharge program on POD 3 led to a biased loss of subjects. Thirty-three per cent of subjects were lost or discharged on POD 3, usually to a rehabilitation facility. Discharged subjects were significantly older than retained subjects. Ages of retained and discharged subjects were $63.8 \pm 8.8$ years and $69.2 \pm 9.4$ years, respectively. In practical terms, this meant that when questionnaire data were manually collected in the afternoon on POD 2, they reflected $4 \mathrm{~h}$ to $7 \mathrm{~h}$ of oral opioid use. Because data on opioid and sideeffect drug use were gathered retrospectively from electronic medication records up to $24: 00$ on POD 2, these outcomes reflected a longer period of oral opioid use (12 h to $15 \mathrm{~h}$ ), but was still less than $24 \mathrm{~h}$.

The present study is nonetheless noteworthy for several reasons. First, it adds to the limited literature on PCOA. Although PCOA has been in use for years at some institutions, it does not often appear in reviews of PCA techniques (13-15),

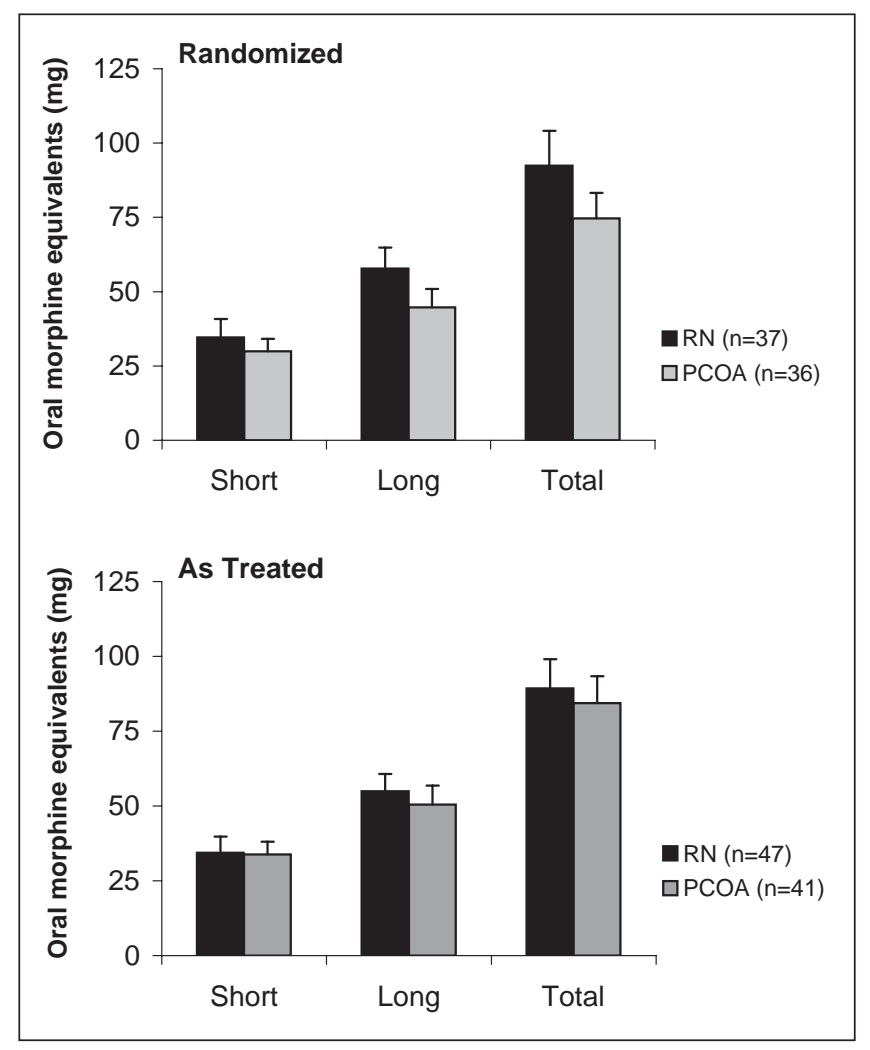

Figure 3) Subjects' total short-and long-acting oral opioid use on postoperative day 2. PCOA Patient-controlled oral analgesia group; RN Nurse-administered oral analgesia group

and its efficacy has only been formally evaluated in a limited number of studies using specialized delivery systems $(7,8)$. Second, the present study assessed a much simpler system (child-resistant, bedside vial containing a single dose) for PCOA administration. Third, although not statistically significant, we observed that PCOA subjects tended to use fewer oral opioids and fewer medications for two opioid side effects (pruritis and constipation). These observations suggest that future studies on PCOA should track these particular outcomes, with the proviso that it would be preferable to track side effects directly. The fact that PCOA subjects did not use more opioids is also consistent with earlier demonstrations that patients do not take more opioids when administered IV PCA compared with traditional RN-administered analgesia (16).

It would also be helpful for future studies to explore the merits of PCOA for postoperative pain with larger sample sizes and for a longer study period. Future studies may also explore the efficacy and safety of using long- and short-acting oral opioids for postoperative pain control. Although the current practice is to provide both long- and short-acting opioids for postoperative pain control, this practice may be analogous to the provision of a basal opioid infusion with IV PCA - a practice that significantly increases the rate of adverse effects without making a difference in analgesic quality (17-19).

\section{CONCLUSION}

Although the present study did not find significant differences between PCOA and RN-administered oral analgesia, we continue to expand the use of PCOA in our facility because it is 
in keeping with a patient-centred care philosophy (20). Specifically, we believe that PCOA enables patients to extend their sense of control from IV PCA into the realm of oral analgesia. PCOA is also a simple, low-cost system that teaches patients how and when to take analgesic tablets for pain control before they are sent home with a pain prescription to take 'as needed'. We recognize that further research is necessary to validate its role.

ACKNOWLEDGEMENTS: The present study was supported by grants from Krembil Nursing Awards, University Health Network, The Canadian Nurses Foundation and the Nursing Care Partnership Program.

\section{REFERENCES}

1. Apfelbaum JL, Chen C, Mehta SS, Gan TJ. Postoperative pain experience: Results from a national survey suggest postoperative pain continues to be undermanaged. Anesth Analg 2003;97:534-40.

2. Coley KC, Williams BA, DaPos SV, Chen C, Smith RB. Retrospective evaluation of unanticipated admissions and readmissions after same day surgery and associated costs. J Clin Anesth 2002;14:349-53.

3. Watt-Watson J, Garfinkel P, Gallop R, Stevens B, Streiner D. The impact of nurses' empathic responses on patients' pain management in acute care. Nurs Res 2000;49:191-200.

4. Watt-Watson J, Stevens B, Costello J, Katz J, Reid G. Impact of preoperative education on pain management outcomes after coronary artery bypass graft surgery: A pilot. Can J Nurs Res 2000;31:41-56.

5. Trueblood A, Manning DW. Analgesia following total knee arthroplasty. Curr Opin Orthop 2007;18:76-80.

6. Hudcova J, McNicol E, Quah C, Lau J, Carr DB. Patient controlled opioid analgesia versus conventional opioid analgesia for postoperative pain. Cochrane Database Syst Rev 2006; (4):CD003348.

7. Striebel HW, Romer M, Kopf A, Schwagmeier R. Patient controlled oral analgesia with morphine. Can J Anaesth 1996;43:749-53.

8. Striebel HW, Scheitza W, Philippi W, Behrens U, Toussaint S. Quantifying oral analgesic use using a novel method and comparison with patient-controlled intravenous analgesic use. Anesth Analg 1998;86:1051-3.

9. Kastanias P, Snaith K, Robinson S. Patient-controlled oral analgesia: A low-tech solution in a high-tech world. Pain Manag Nurs 2006;7:126-32.

10. Su L, Tucker R, Frey SE, et al. Comparison of pain measures in surgical patients. J Nurs Meas 1999;7:135-52.

11. Tittle MB, McMillan SC, Hagan S. Validating the brief pain inventory for use with surgical patients with cancer. Oncol Nurs Forum 2003;30:325-30.

12. Carlson J, Youngblood R, Dalton J, Blau W, Lindley C. Is patient satisfaction a legitimate outcome of pain management? J Pain Symptom Manage 2003;25:264-75.

13. Grass JA. Patient-controlled analgesia. Anesth Analg 2005;101(5 Suppl):S44-61.

14. Lehmann KA. Recent developments in patient-controlled analgesia. J Pain Symptom Manage 2005;29(5 Suppl):S72-89.

15. Viscusi ER. Patient-controlled drug delivery for acute postoperative pain management: A review of current and emerging technologies. Reg Anesth Pain Med 2008;33:146-58.

16. Ballantyne J, Carr D, Chalmers T, Dear K, Angelillo I, Mosteller F. Postoperative patient-controlled analgesia: Meta-analyses of initial randomized control trials. J Clin Anesth 1993;5:182-93.

17. Principles of Analgesic Use in the Treatment of Acute Pain and Cancer Pain, 5th edn. Glenview: American Pain Society, 2003.

18. Hagle M. Respiratory depression in adult patients with intravenous patient-controlled analgesia. Orthop Nurs 2004;23:18-29.

19. Weber L. Implementation of standard order sets for patientcontrolled analgesia. Am J Health Sys Pharm 2008;65:1184-91.

20. Gerteis M, Edgeman-Levitan S, Daley J, Delbanco TL. Through the Patients' Eyes: Understanding and Promoting Patient-centered Care. San Francisco: Jossey-Bass, 1993. 


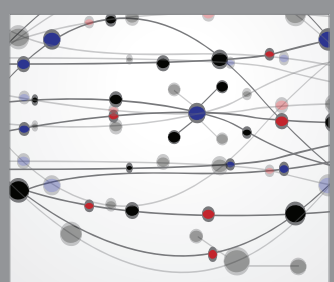

The Scientific World Journal
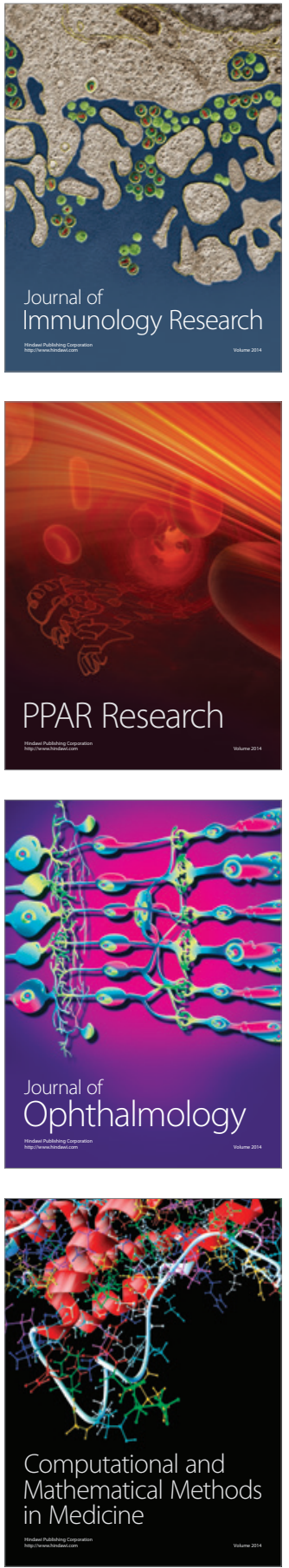

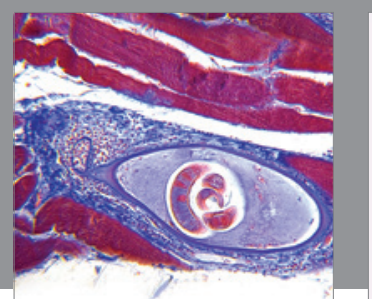

Gastroenterology Research and Practice

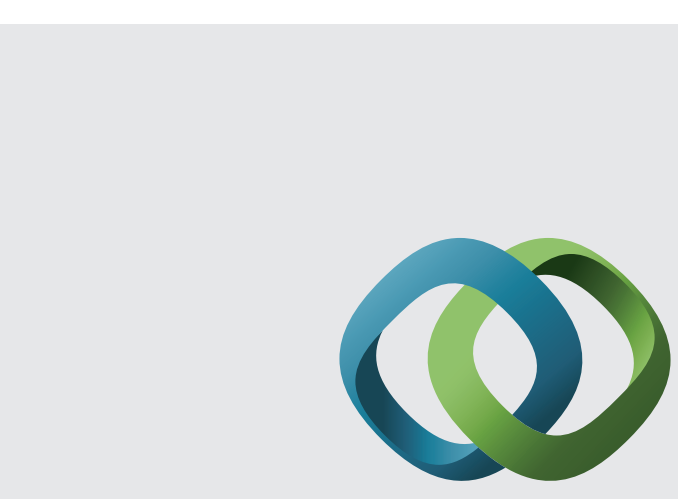

\section{Hindawi}

Submit your manuscripts at

http://www.hindawi.com
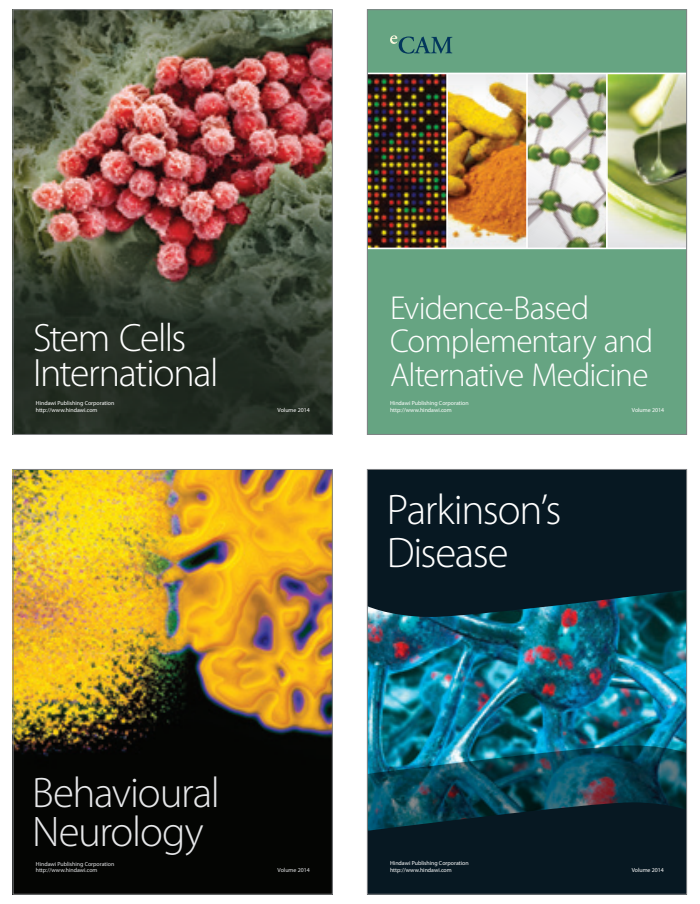
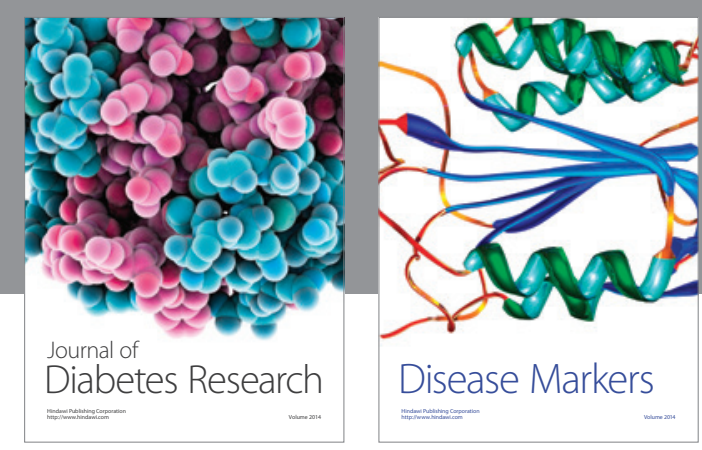

Disease Markers
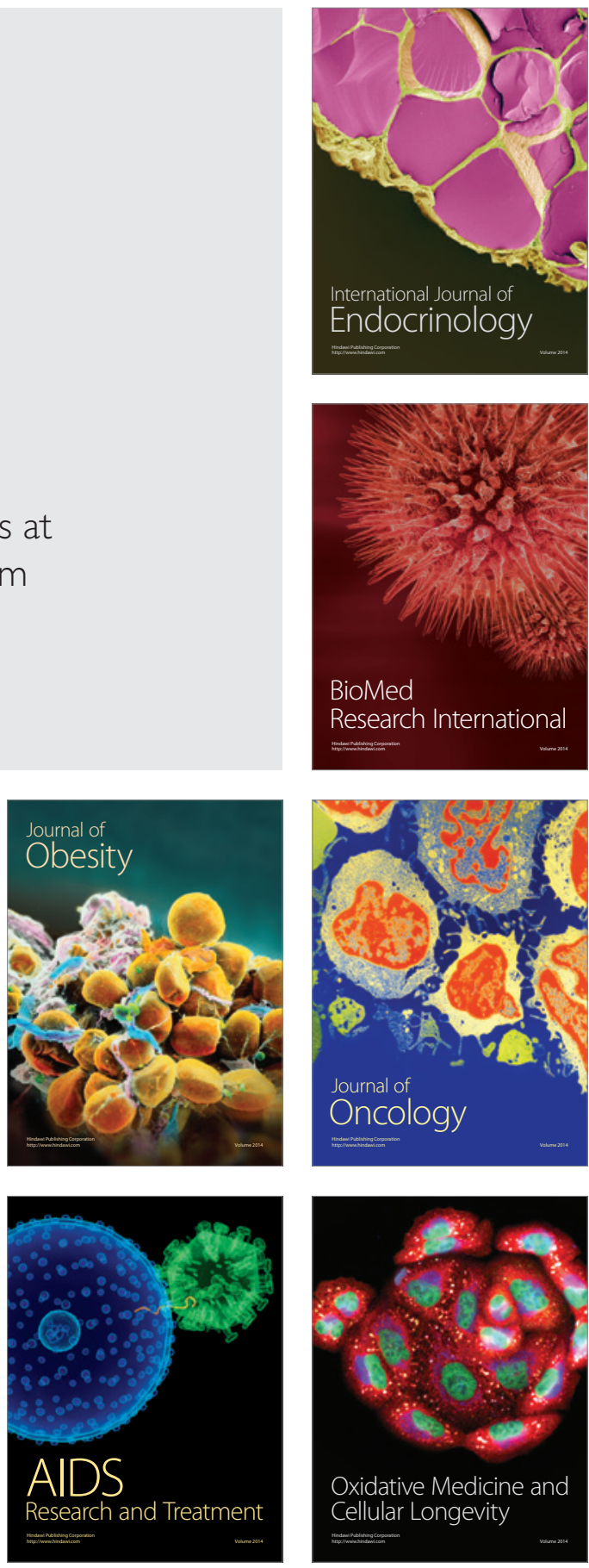